\title{
Prescribing patterns of antibiotics for children before admission to a paediatric ward in Jaffna Teaching Hospital
}

\author{
S K Arulmoli ${ }^{1}$, S Sivachandiran ${ }^{2}$, B J C Perera ${ }^{3}$ \\ Sri Lanka Journal of Child Health, 2009; 38: 121-123
}

(Key words: Prescribing pattern of antibiotics, children)

\begin{abstract}
Antibiotics are commonly prescribed drugs in paediatrics. However, the threat of antibiotic resistance among children is a cause for concern. A study of the administration patterns of antibiotics prior to admission was carried out on children admitted to a paediatric ward of Teaching Hospital, Jaffna from June to August 2008, using a pre-tested structured questionnaire. Descriptive and basic statistical tests were used to analyse the data. The total number of admissions to the ward was 420 out of which 227 (54\%) had been given antibiotics prior to admission. Of this, $53(23 \%)$ were infants. Of the entire cohort, oral antibiotics were given to 214 $(94 \%)$ and $47(22 \%)$ of them were given two or more antibiotics. Amoxicillin (48\%), erythromycin (20\%) and cephalexin (16\%) were the antibiotics commonly prescribed. Sixty three percent were prescribed antibiotics by general practitioners and $16 \%$ were given antibiotics without consulting a doctor. Only $53(23 \%)$ of the parents knew the name and the sideeffects of the antibiotics used on the children. Hospital stay was significantly more for children given prior antibiotics than for those who did not have prior antibiotics $(14 \%$ against $8 \% \mathrm{p}<0.05)$. Other medications had been administered to 298 $(71 \%)$. In order to reduce the risk of antibiotic resistance of microbes, an antibiotic policy should be carefully instituted and implemented.
\end{abstract}

\section{Introduction}

Infants and children are among the most vulnerable population groups that contract illnesses. The use of antimicrobial agents has become a routine practice for the treatment of paediatric illnesses ${ }^{1,2}$ and

${ }^{1}$ Acting Paediatrician, ${ }^{2}$ Biometrician, Teaching Hospital, Jaffna, ${ }^{3}$ Consultant Paediatrician, Colombo.

(Received on 18 December 2008. Accepted on 29 January 2009) antibiotics are among the most commonly prescribed drugs in paediatrics. Although the key role played by antibiotics in the treatment of infectious diseases in developing countries should be acknowledged, there are reports of irrational use of antibiotics ${ }^{3,4}$ which may even lead to infections that are worse than those caused originally. Paediatricians and other medical personnel who provide health care for infants and children in developing countries are faced with numerous challenges due to the shortage of appropriate drugs, costs and lack of infrastructure. The rising incidence of bacterial resistance to commonly used antibiotics, particularly the emergence of multi-drug resistant organisms has made it mandatory that antibiotics are used judiciously in paediatric practice. Many of the antibiotics are unnecessarily prescribed for viral infections such as the common cold. It has been reported that $20-50 \%$ of antimicrobial usage is questionable or inappropriate ${ }^{5}$.

Several professional societies have issued guidelines designed to reduce the use of antibiotics worldwide by means of various control strategies ${ }^{6,7}$. Detailed knowledge of antibiotic prescription pattern is important before policies and measures can be implemented. Appropriate drug utilization studies are essential to assess whether drugs are correctly used in terms of medical, social and economic aspects.

A similar trend of inappropriate usage of antibiotics was observed in Jaffna. A significant majority of children are administered antibiotics before admission to hospital. There is no published study on this aspect and the present assessment was carried out in Ward 11 of the Teaching Hospital, Jaffna to address this deficiency.

\section{Objectives}

- To assess the prescribing patterns of antibiotics prior to admission in children who were admitted to Teaching Hospital, Jaffna. 
- To determine the levels of parental knowledge regarding antibiotics.

\section{Method}

A survey was carried out on the parents or guardians of children admitted to Paediatric Ward 11, Teaching Hospital, Jaffna in June, July and August 2008 using a pre-tested structured questionnaire to assess the antibiotic prescribing pattern prior to admission. Basic demographic information and details of the prescribed antibiotics and their prescribing patterns together with details of other medications, clinical presentation, hospital diagnosis, duration of hospital stay and parental knowledge regarding antibiotics were documented in all patients admitted to the ward. Data was analyzed using the statistical package for social sciences (SPSS) computer software programme.

\section{Results}

A total of 420 children was admitted to Ward 11 of the Teaching Hospital, Jaffna during the study period. The gender and age distributions of the children admitted to the hospital are given in Figures 1 and 2 .

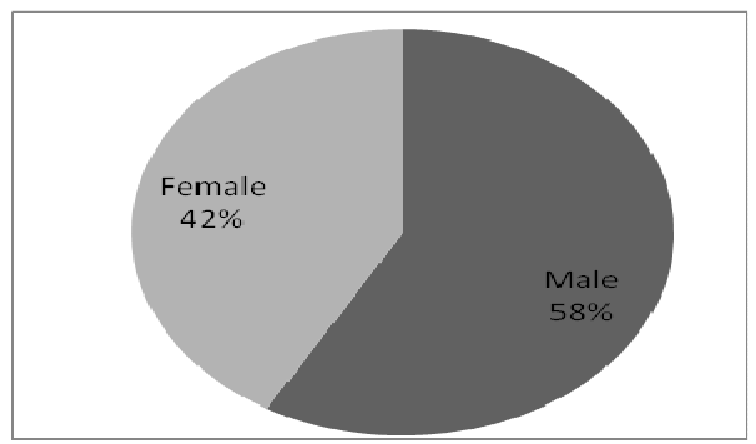

Figure 1: Gender Distribution

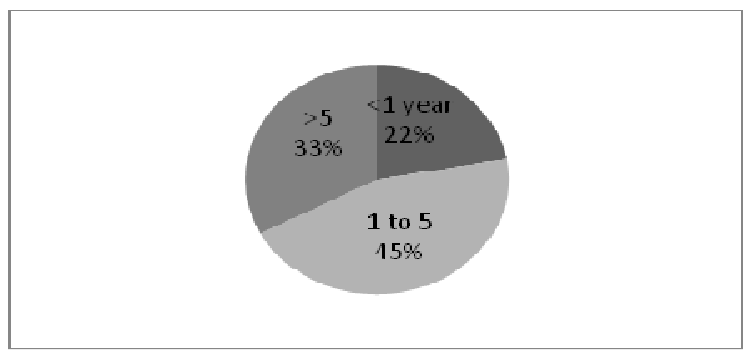

Figure 2: Age Distribution

The children admitted from different geographical divisions of Jaffna are given in figure 3 . Highest number $(75 \%)$ was from Valigamam and $11 \%$ were from the islands.

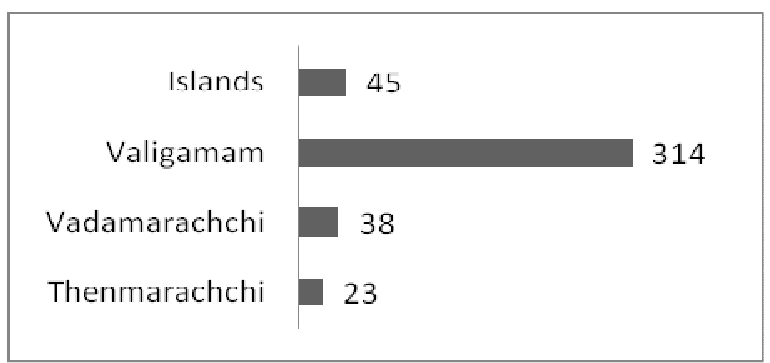

Figure 3: No. of children admitted from different areas

Percentages of children given antibiotics before admission with their ages are shown in Figure 4.

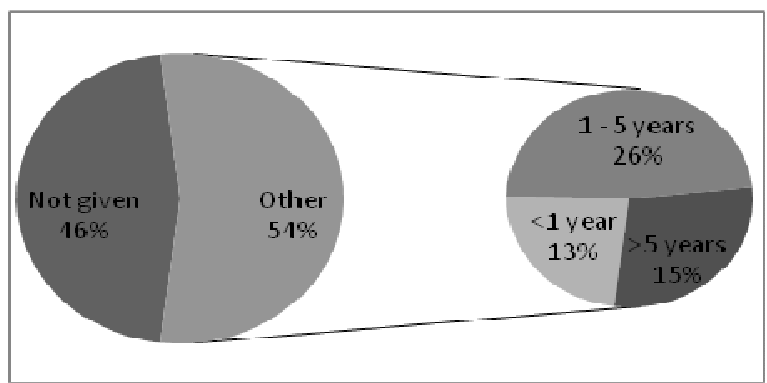

Figure 4: Percentage of children given antibiotics before admission with their ages

Several antibiotics had been given to these children. A significant proportion, 110 (48\%), had amoxicillin, $46(20 \%)$ were given erythromycin, $36(16 \%)$ were given cephalexin and $17(7 \%)$ were given cotrimoxazole. Majority of the antibiotics viz. 144 $(63 \%)$ were prescribed by general practitioners. Only $11(5 \%)$ had been prescribed antibiotics by a paediatrician. A significant number viz. 36 (16\%) was given antibiotics without consulting a doctor.

Numbers of children given antibiotics for different diagnoses prior to admission with their ages are shown in Figure 5.

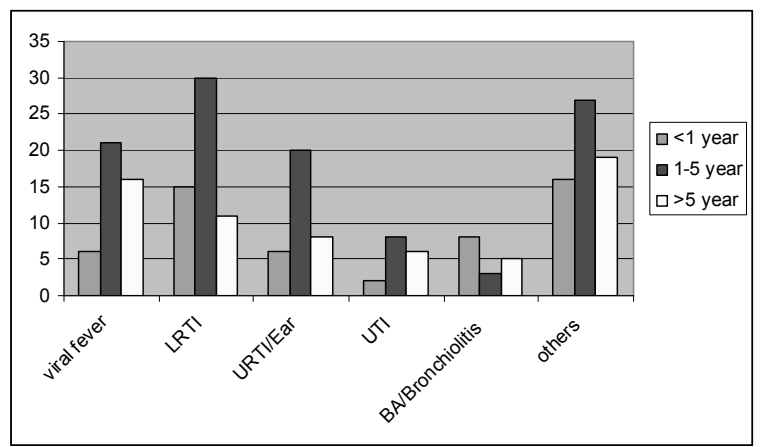

Figure 5: Numbers of children given antibiotics for different diagnoses prior to admission with their ages 
Twenty five percent of children were prescribed antibiotics for lower respiratory tract infections (LRTIs), $19 \%$ for viral fever and $14 \%$ for upper respiratory tract infections (URTIs) / ear infections. About 7\% were prescribed antibacterials for bronchial asthma / bronchiolitis and 7\% had been given antibiotics for urinary tract infections.

Figure 6 shows the duration of hospital stay of children who had been given or not given antibiotics before admission to the ward.

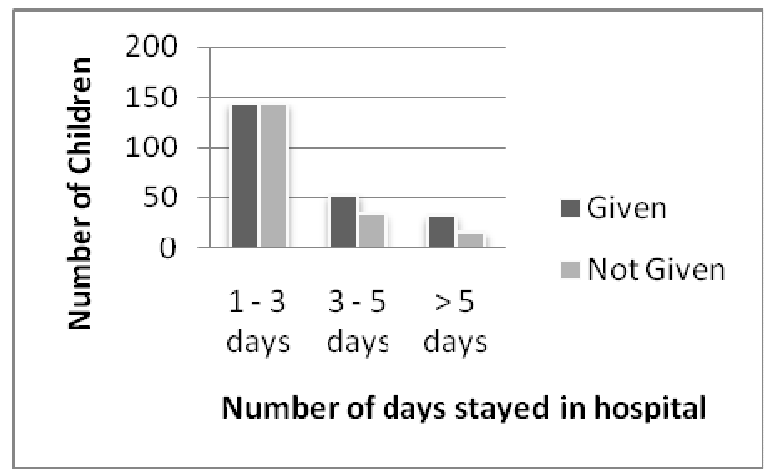

Figure 6: Hospital stay of children given or not given antibiotics before admission

\section{Discussion}

The total number of patients admitted to the ward was 420 . The admission numbers from different areas (Figure 3) can be correlated to the population distribution of the divisions and the distance from the teaching hospital. The Valligamam division has the highest population and the hospital is situated in the Valligamam division. Majority ( $82 \%$ ) of the patients were admitted via the outpatient department.

Of the 420 children surveyed, 227 (54\%) were given antibiotics before admission to the hospital (figure 4). Of this total, $23 \%$ were below one year, $48 \%$ between 1 to 5 years and the rest above five years of age (Figure 4). Most (94\%) children were given oral antibiotics and $22 \%$ were given more than one antibiotic. Amoxicillin (48\%), erythromycin (20\%) and cephalexin $(16 \%)$ were found to be the most frequently used antibiotics. The prescription of antibiotics was mainly based on clinical judgment. A study in $\mathrm{Nepal}^{8}$ too showed the same pattern of antibiotic usage among children.

Figure 5 clearly indicates that the antibiotics were not appropriately prescribed. Higher numbers of children were given antibiotics for diseases like viral fever, URTI, LRTI etc. This suggests that the antibiotics were given without a definitive clinical diagnosis.
Sixty three percent of children were prescribed antibiotics by the general practitioner. About $16 \%$ were given antibiotics without consulting a doctor. The number of days stayed in hospital (figure 6) was significantly less $(p<0.05)$ for children who had not been given antibiotics before admission.

\section{Conclusions}

- Prescription patterns and usage of antibiotics in this study was inappropriate.

- Parental knowledge on antibiotics was inadequate.

\section{References}

1. Sanz EJ, Bergman U, Dahlstorm M. Paediatric drug prescribing. European Journal of Clinical Pharmacology 1989; 37:65-8.

2. Summers RS, Summers B. Drug prescribing in paediatrics. Annals of Tropical Paediatrics 1986; 6:129-33.

3. Pricipi $\mathrm{N}$ et al. Control of antibiotic therapy in paediatric patients. Developmental Pharmacology and Therapeutics 1981; 2:145-55.

4. Schllenberg E, Albritton WL. Antibiotic misuse in a paediatric teaching hospital. Canadian Medical Association Journal 1980; 122:49-52.

5. Hecker MT, Aron DC, Patel NP, Lehmann MK. Unnecessary use of antimicrobials in hospitalized Patient. Archives of Internal Medicine 2003; 163:972-8.

6. Kolar J, Kadakova E. Prescription of antimicrobial drugs to hospitalized children. Annals of Pharmacotherapy 1993; 26:974-7.

7. Marr JJ, Moffet HL, Kunin CM. Guidelines for improving the use of antimicrobial agents in hospitals: a statement by the Infectious Diseases Society of America. Journal of Infectious Diseases 1988; 157:869-76.

8. Palikhe N. Prescribing pattern of antibiotics in paediatric hospital of Kathmandu Valley. Journal of Nepal Health Research Council 2004; 2: 31-6. 